\title{
A Novel Integrated (OF-HC-EPM) Approach to Study Anxiety Related Depressive Behavior in Mice Model: A Comparison of Neuro Standards
}

\author{
Mohammad M. Billah", ${ }^{1, *}$, Md. A. Rayhan ${ }^{2}$, Syed A. Yousuf ${ }^{3}$, Kashfia Nawrin' ${ }^{1}$, Johir Rayhan ${ }^{4}$, \\ Elmabruk M. Khengari ${ }^{5}$ \\ ${ }^{1}$ Department of Pharmacology, Institute for Pharmaceutical Skill Development and Research, Bangladesh \\ ${ }^{2}$ Department of Genetic Engineering and Biotechnology, East West University, Bangladesh \\ ${ }^{3}$ Department of Anesthesiology, Bangabandhu Sheikh Mujib Medical University, Bangladesh \\ ${ }^{4}$ Department of Pharmacy, Jahangirnagar University, Bangladesh \\ ${ }^{5}$ Tripoli Children Hospital, Libya
}

Copyright $\odot 2019$ by authors, all rights reserved. Authors agree that this article remains permanently open access under the terms of the Creative Commons Attribution License 4.0 International License

\begin{abstract}
Open Field, Hole Cross and EPM are three widely acceptable experimental methods used to evaluate sedative-anxiolytic potential. The theories behind introducing these fields were to challenge the rodents to a novel environment. However, the behavioral changes caused by these environments often get influenced by rodent's identical neurologic conditions. The major challenges faced by the researchers are variations due to first administration against repeated administration, utilizing same rodent for another experiment but in different time or using different rodent for different experiments. Keeping the drawbacks in consideration, the present study undertook a newly modified (OF-HC-EPM) approach to integrate the experimental fields so as to utilize the same rodents with single oral administration for exposure to different fields which had allowed nullifying the risk of individual and time dependent variance. Anxiolytics, atypical antidepressants, selective serotonin reuptake inhibitors, serotonin-norepinephrine reuptake inhibitors, tricyclic antidepressants as well as the combined anxiolytics and antidepressants were administered to Swiss Albino Mice and their respective behavioral changes were observed. The new approach proved to be an essential tool for evaluating neuropharmacological potentials.
\end{abstract}

Keywords Open Field, Hole Cross, Elevated Plus Maze, Square Cross, Grooming, Rearing, Stretch Attend Posture, Freezing

\section{Introduction}

Depression followed by anxiety is the common phenomenological symptoms in neurobehavioral study, though rarely co-investigated within a particular experimental model. A range of test methods have been developed to evaluate these two neurological conditions separately but few approaches have been reported to understand the correlation. The major drawbacks in such study designs are the limit to challenge the same subject keeping the same physiological, psychological and surrounding environmental conditions for different experiments - the reason that drives the investigators to design the experiments individually with identical subjects. As a consequence, major gaps on relevancy in behavior are observed. Thus, the present study was aimed to minimize this gap by integrating three experimental designs in a cascade for utilizing the same subjects with their unique characteristics. Furthermore, a comparative study of neuro standards was incorporated into the present design to evaluate the validity of this new approach.

Open Field, Hole Cross and Elevated Plus Maze (EPM) are three most widely used methods for evaluating neurological condition of rodents. The elicitation of rodent's behavior in these fields are dependent upon many test factors like strain, age, sex; prior test experience, presence of stimuli/inducer in test apparatus, forced feeding and handling by experimenter [1,2]. Open field was originally developed for assessing comparative psychology of rodents however it is now also used for determination of altered exploration, anxiety and emotionality [3]. Hole Cross apparatus was introduced by Takagi et al. for measurement of spontaneous movement of rodents [4]. On the other hand, EPM was designed for the investigation of anxiety related behavior [5].

Neurological conditions like anxiety and depression are treated with different classes of neuro drugs, sometimes in 
their combinations. For the present study, drugs from the group of anxiolytics, atypical antidepressants, selective serotonin reuptake inhibitors, serotonin-norepinephrine reuptake inhibitors, tricyclic antidepressants as well as the combined anxiolytics and antidepressants were used. To distinguish the common behavioral features between sedation and depression, drugs from different classes were considered for this study.

\section{Materials and Methods}

\subsection{Drugs and Chemicals}

Diazepam (Square Pharmaceuticals Ltd.), Mirtazapine (Sun Pharmaceuticals Ltd.), Citalopram (Incepta Pharmaceuticals Ltd.), Duloxetine (Incepta Pharmaceuticals Ltd.), Imipramine (Sandoz), Flupentixol \& Melitracen (Beximco Pharmaceuticals Ltd.), Fluphenazine \& Nortriptyline (Aristopharma) were obtained.

\subsection{Experimental Animal}

Swiss Albino mice (Sex: Female, Age: 45 days, Weight: 28-33g) were selected for all groups of the present study. Mice were habituated with a $12 \mathrm{~h} \mathrm{light/dark} \mathrm{cycle,} \mathrm{ambient}$ temperature, air ventilation and ad libitum food and water at animal house of Institute of Pharmaceutical Skill Development and Research. Nine groups $(\mathrm{N}=5)$ were formed intended to be challenged after oral gavage with subsequent agents as Group 1: Control (No gavage), Group 2: Blank (Water), Group 3: Diazepam ( $1 \mathrm{mg} / \mathrm{kg}$ ), Group 4: Mirtazapine (3mg/kg), Group 5: Citalopram $(4 \mathrm{mg} / \mathrm{kg})$, Group 6: Duloxetine $(4 \mathrm{mg} / \mathrm{kg})$, Group 7: Imipramine $(5 \mathrm{mg} / \mathrm{kg})$, Group 8: Flupentixol $(0.1 \mathrm{mg} / \mathrm{kg})+$ Melitracen $(2 \mathrm{mg} / \mathrm{kg})$, Group 9: Fluphenazine $(0.1 \mathrm{mg} / \mathrm{kg}) \quad+$ Nortriptyline $(2 \mathrm{mg} / \mathrm{kg})$.

\subsection{Experimental Design (The OF-HC-EPM Integration)}

A cascade of novelty induced challenge has been executed by integrating open field, hole cross and elevated plus maze fields (OF-HC-EPM Integration Approach). After oral gavage, immediately each animal was placed on open field for 3 minutes, shifted to the hole cross apparatus for next 3 minutes (however for simplifying, the time denoted as $0 \mathrm{~min}$ for hole cross) and finally transferred to EPM apparatus for last 3 minutes (the time denoted as 0 min for EPM), each having a total 9 minutes of challenge. This cascade for behavioral evaluation was repeated in 30 , 60,90 and 120 minutes after oral feeding. The animal was allowed to calm for around 20 minutes before next interval.

\subsubsection{Open Field Test}

An Open Field apparatus has a dimension of $60 \times 60 \times 60$ $\mathrm{cm}$, floor of which is marked as square boxes $(5 \times 5 \mathrm{~cm})$ alternatively colored black and white [6,7]. At a 3-minute interval, mice were observed for parameters such as number of square crossed, number of center square crossed and duration, Grooming, Rearing and Freezing [8,9].

\subsubsection{Hole Cross Test}

A Hole Cross apparatus $(30 \times 20 \times 14 \mathrm{~cm})$ has a middle partition which divides it into two equal compartments (each $15 \times 10 \times 14 \mathrm{~cm}$ ) in which there is a $3 \mathrm{~cm}$ hole at height of $7 \mathrm{~cm}$ [10]. At each 3-minute interval, mice were allowed to freely move from one compartment to another through the hole. The number of holes crossed was measured during the observation [11].

\subsection{3 .Elevated Plus Maze Test}

An Elevated Plus Maze (EPM) apparatus consists of two opposite open arms and two opposite close arms intersecting in Plus shape. Each arm has a length of $14 \mathrm{~cm}$, width of $5 \mathrm{~cm}$ and the close arm has wall height of $14 \mathrm{~cm}$ [12]. Mice were placed in the apparatus facing the open arm and allowed to freely move in any direction. At each interval, mice were observed for the open and close arm entry and duration [13].

\subsection{Statistical Analysis}

Statistical analysis of data was done by utilizing the method of one-way analysis of variance (ANOVA) followed by Dunnett's t tests using SPSS 25 for windows. The results obtained were compared with the control group. $\mathrm{P}$ values $<0.05,0.01$ and 0.001 were considered statistically significant.

\section{Results}

\subsection{Open Field Test}

\subsubsection{Square Crossing/Line Crossing}

Maximum number of square crossing was observed by imipramine (159.4) immediately after the administration (Figure 1). However, citalopram showed consistency in increasing the number. On the other hand, minimum activity was observed by the combination of fluphenazine and nortriptyline (17.0) at $90 \mathrm{~min}$. Duloxetine followed the decreasing pattern in $90 \mathrm{~min}$ (18.8) \& $120 \mathrm{~min}$ (12.2) as well. 


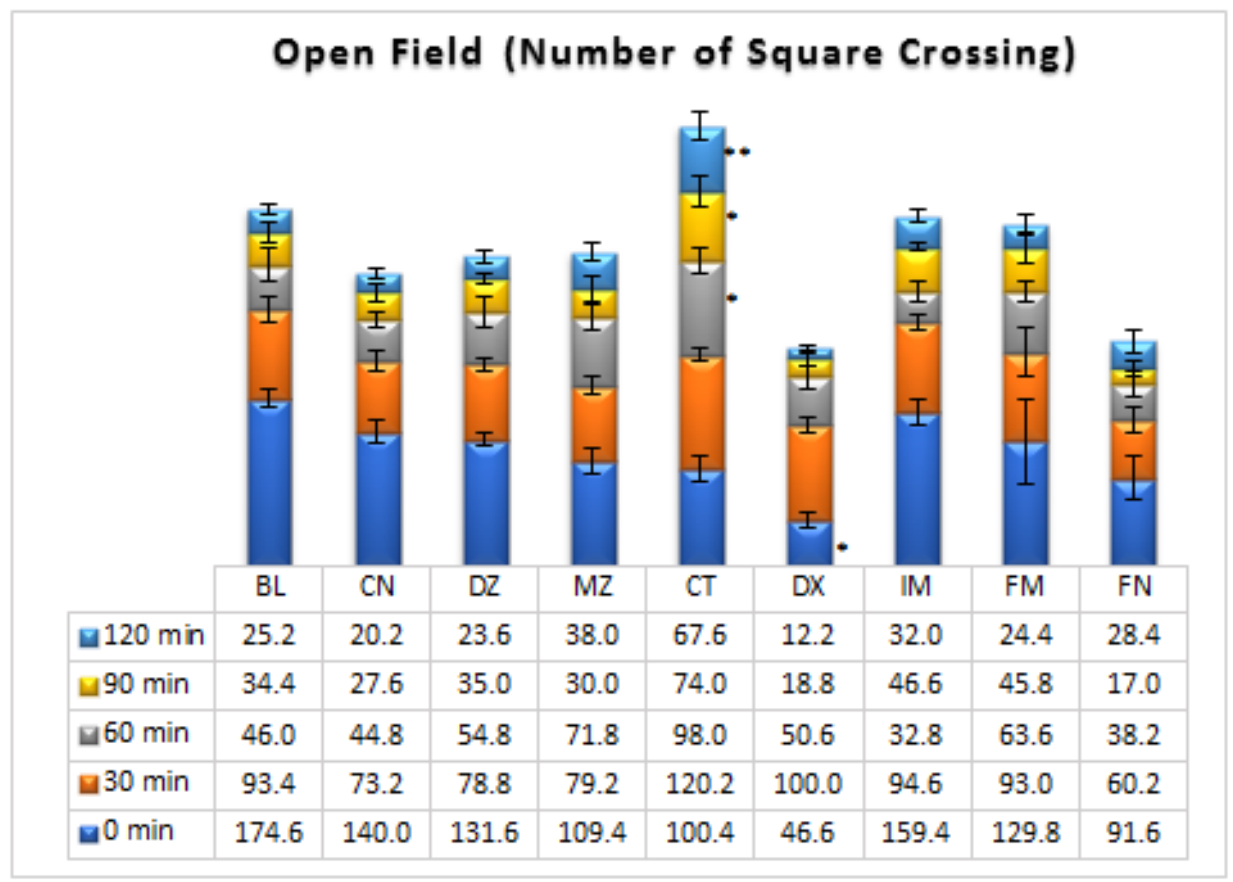

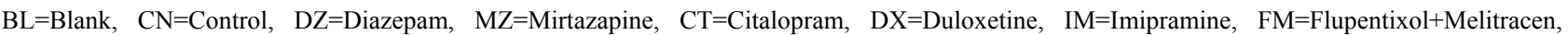
$\mathrm{FN}=$ Fluphenazine+Nortriptyline. Values are expressed as mean \pm S.E.M., $(\mathrm{n}=5) ;{ }^{*} \mathrm{P}<0.05,{ }^{* *} \mathrm{P}<0.01$; Dunnett t-test (2 sided) treated one group as control (water) and compared all other groups against it.

Figure 1. Number of Square crossed by the mice in different time intervals

\subsubsection{Rearing}

Citalopram and imipramine were found with increased rearing (Figure 2). On the contrary, diazepam and mirtazapine at all intervals demonstrated the reducing pattern. Alongside, duloxetine and the two combinations (FM \& FN) showed an initial increase followed by gradual decrease in rearing response.

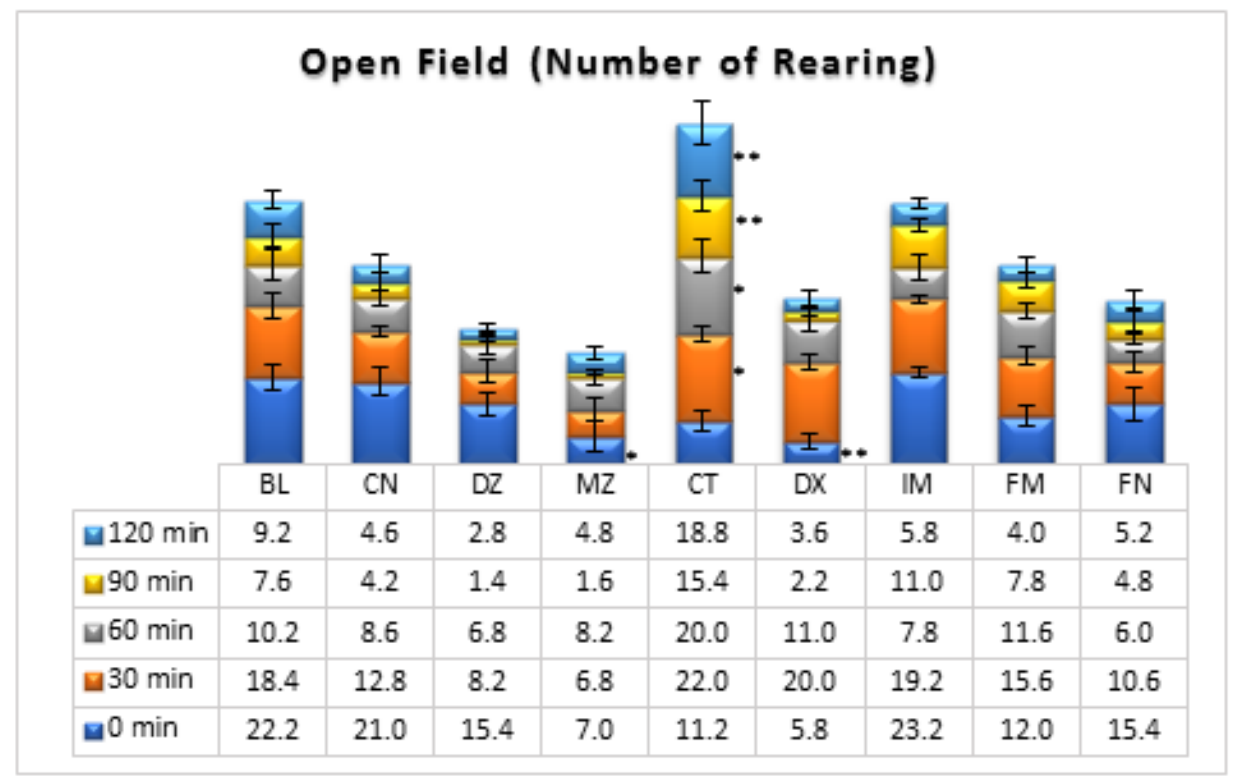

$\mathrm{BL}=$ Blank, $\mathrm{CN}=$ Control, $\mathrm{DZ}=$ Diazepam, $\mathrm{MZ}=$ Mirtazapine, $\mathrm{CT}=$ Citalopram, $\mathrm{DX}=$ Duloxetine, $\mathrm{IM}=$ Imipramine, $\mathrm{FM}=$ Flupentixol + Melitracen, $\mathrm{FN}=$ Fluphenazine+Nortriptyline. Values are expressed as mean \pm S.E.M., $(\mathrm{n}=5) ; * \mathrm{P}<0.05, * * \mathrm{P}<0.01$; Dunnett $\mathrm{t}$-test $(2$ sided) treated one group as control (water) and compared all other groups against it.

Figure 2. Number of Rearing responses exhibited by the mice in different time intervals 


\subsubsection{Grooming}

Grooming response was found to be increased most by diazepam, mirtazapine, imipramine and the two combinations (FM \& FN) compared to control. On the other hand, duloxetine followed by citalopram showed the minimum response in all intervals (Figure 3).

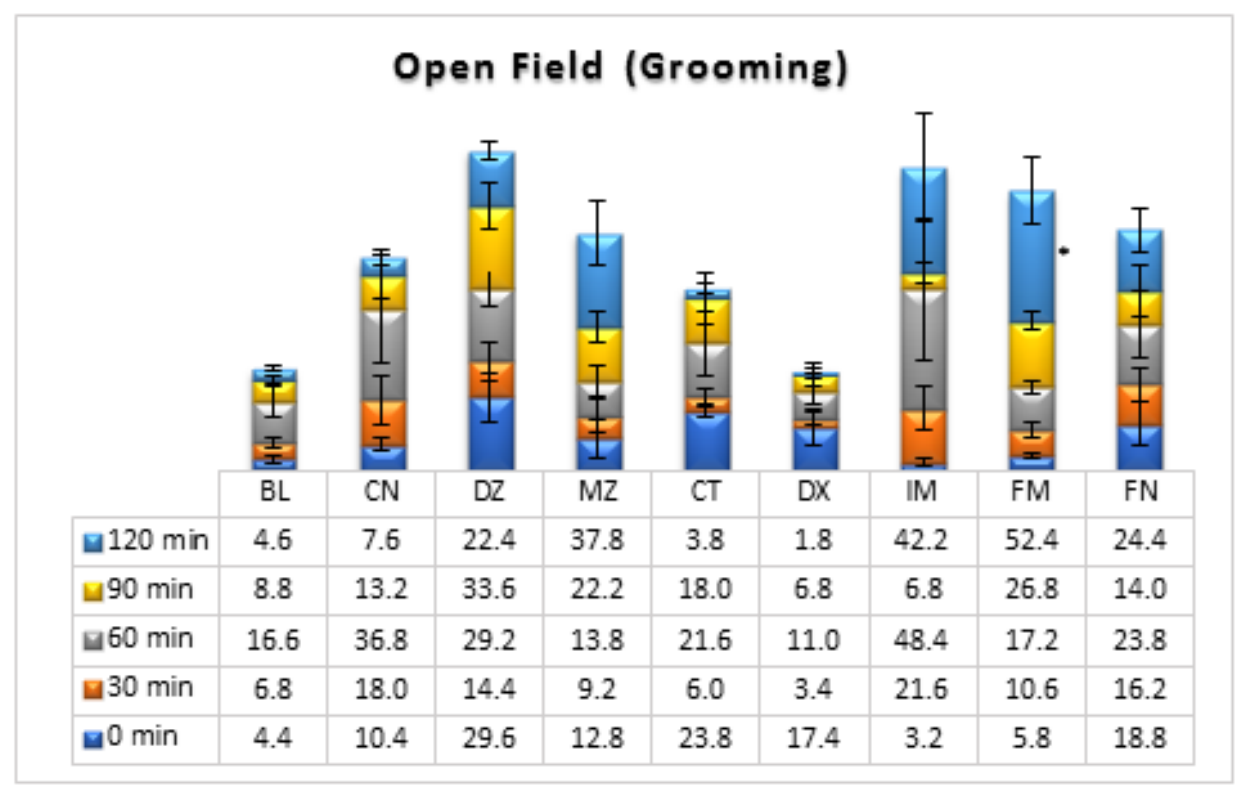

$\mathrm{BL}=$ Blank, $\mathrm{CN}=$ Control, $\mathrm{DZ}=$ Diazepam, $\mathrm{MZ}=$ Mirtazapine, $\mathrm{CT}=$ Citalopram, $\mathrm{DX}=$ Duloxetine, $\mathrm{IM}=$ Imipramine, $\mathrm{FM}=$ Flupentixol + Melitracen, $\mathrm{FN}=$ Fluphenazine+Nortriptyline. Values are expressed as mean \pm S.E.M., $(\mathrm{n}=5) ; * \mathrm{P}<0.05$; Dunnett t-test $(2$ sided) treated one group as control (water) and compared all other groups against it.

Figure 3. Grooming responses exhibited by the mice in different time intervals

\subsubsection{Stretch Attend Posture}

Figure 4 demonstrates the number of stretch attend posture against different standard drugs. The two combinations were found with the highest response whereas diazepam showed the lowest activity for all intervals. Citalopram, duloxetine and mirtazapine followed a common initial increase at $30 \mathrm{~min}$.

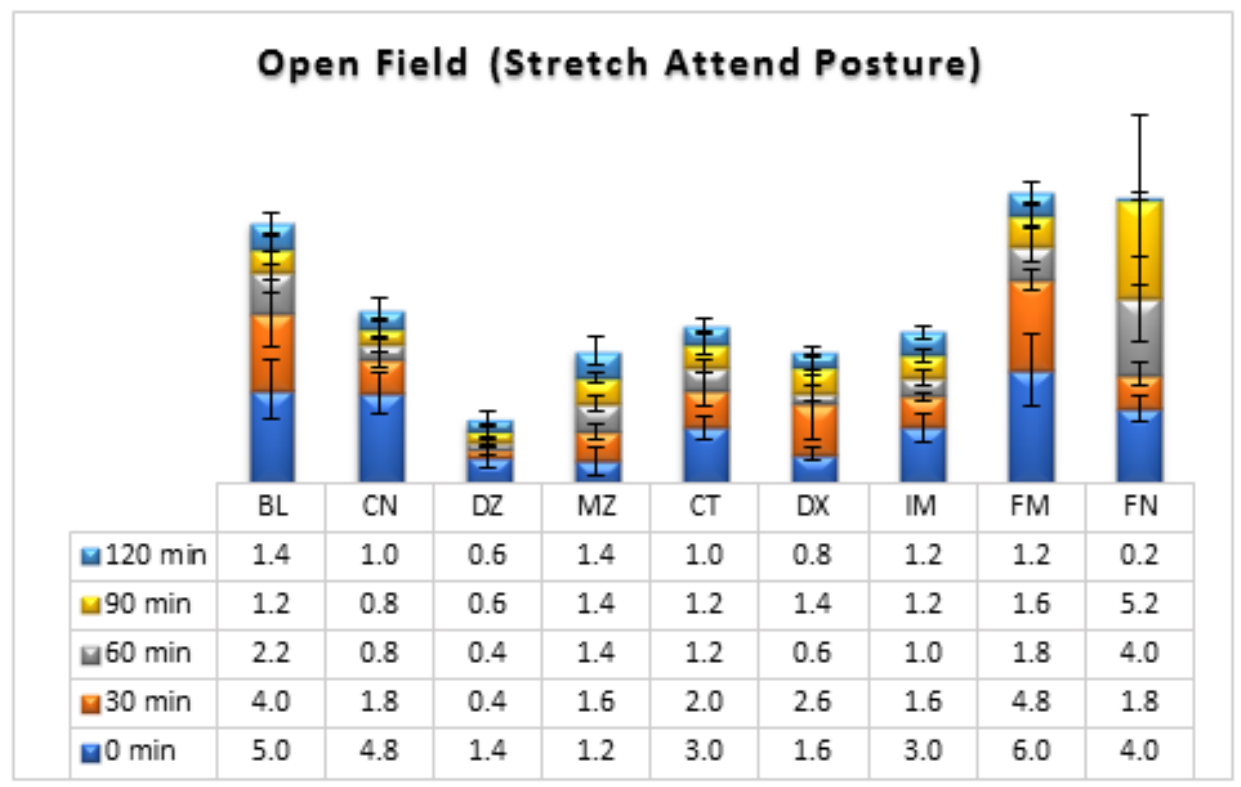

$\mathrm{BL}=$ Blank, $\mathrm{CN}=$ Control, $\mathrm{DZ}=$ Diazepam, $\mathrm{MZ}=$ Mirtazapine, $\mathrm{CT}=$ Citalopram, $\mathrm{DX}=$ Duloxetine, $\mathrm{IM}=\mathrm{Imipramine}, \mathrm{FM}=$ Flupentixol + Melitracen, $\mathrm{FN}=$ Fluphenazine+Nortriptyline. Values are expressed as mean \pm S.E.M., $(\mathrm{n}=5)$; Dunnett $\mathrm{t}$-test $(2$ sided) treated one group as control (water) and compared all other groups against it.

Figure 4. Number of Stretch Attend Posture responses exhibited by the mice in different time intervals 


\subsubsection{Freezing}

Diazepam showed the gradual rise in freezing from $0 \min (11.6)$ and continued to $120 \min (61.0)$. Maximum rise was showed by the combination of fluphenazine and nortriptyline at $90 \mathrm{~min}$ (87.0) and duloxetine at $120 \mathrm{~min}$ (82.2). All drugs showed a common increase from $60 \mathrm{~min}$ to $120 \mathrm{~min}$. Lowest freezing response was observed by citalopram and imipramine at all intervals (Figure 5).

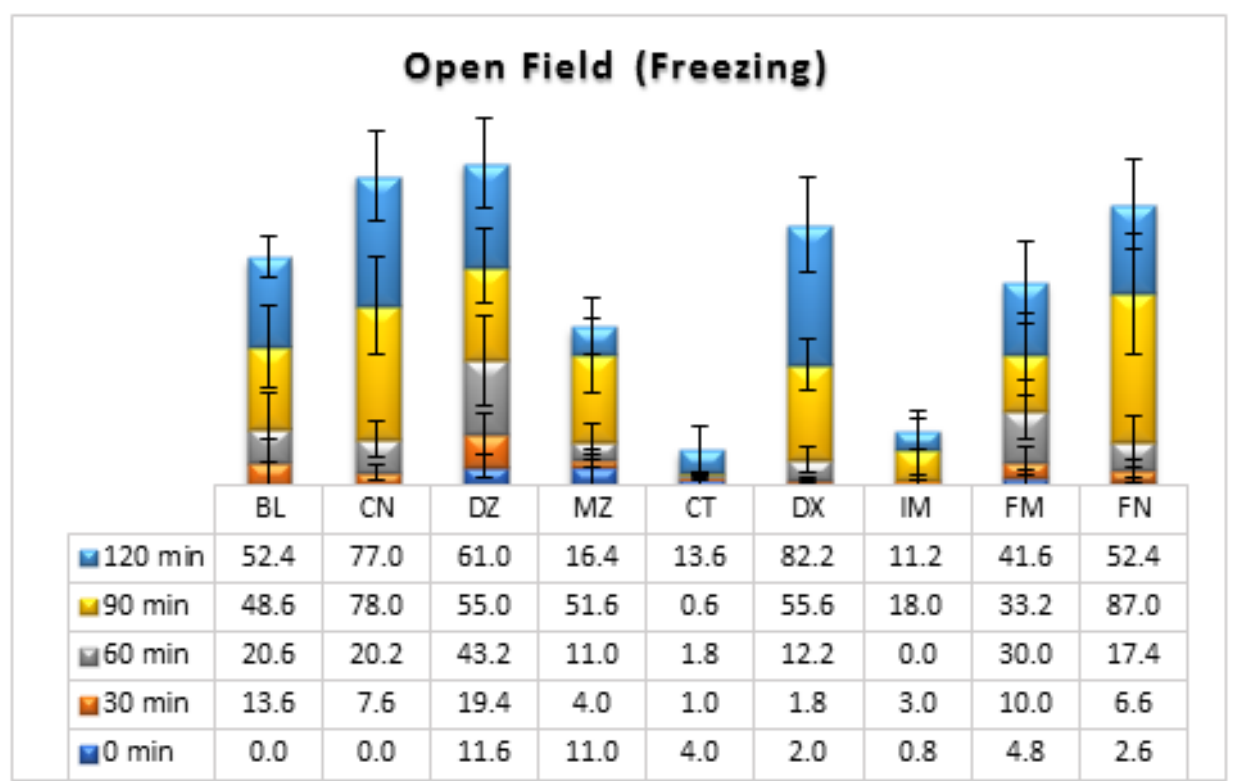

$\mathrm{BL}=$ Blank, $\mathrm{CN}=$ Control, $\mathrm{DZ}=$ Diazepam, $\mathrm{MZ}=$ Mirtazapine, $\mathrm{CT}=$ Citalopram, $\mathrm{DX}=$ Duloxetine, $\mathrm{IM}=$ Imipramine, $\mathrm{FM}=\mathrm{Flupentixol}+\mathrm{Melitracen}$, $\mathrm{FN}=$ Fluphenazine+Nortriptyline. Values are expressed as mean \pm S.E.M., $(\mathrm{n}=5)$; Dunnett $\mathrm{t}$-test $(2$ sided) treated one group as control (water) and compared all other groups against it.

Figure 5. Freezing responses exhibited by the mice in different time intervals

\subsubsection{Center Square Crossing}

Citalopram found with a gradual increase in the number of center square crossing (Figure 6) and duration spent in central squares (Figure 7) from $30 \mathrm{~min}$ to $120 \mathrm{~min}$. Alongside, the two combinations were found to prolong the duration. However, duloxetine failed to show this response at all intervals.

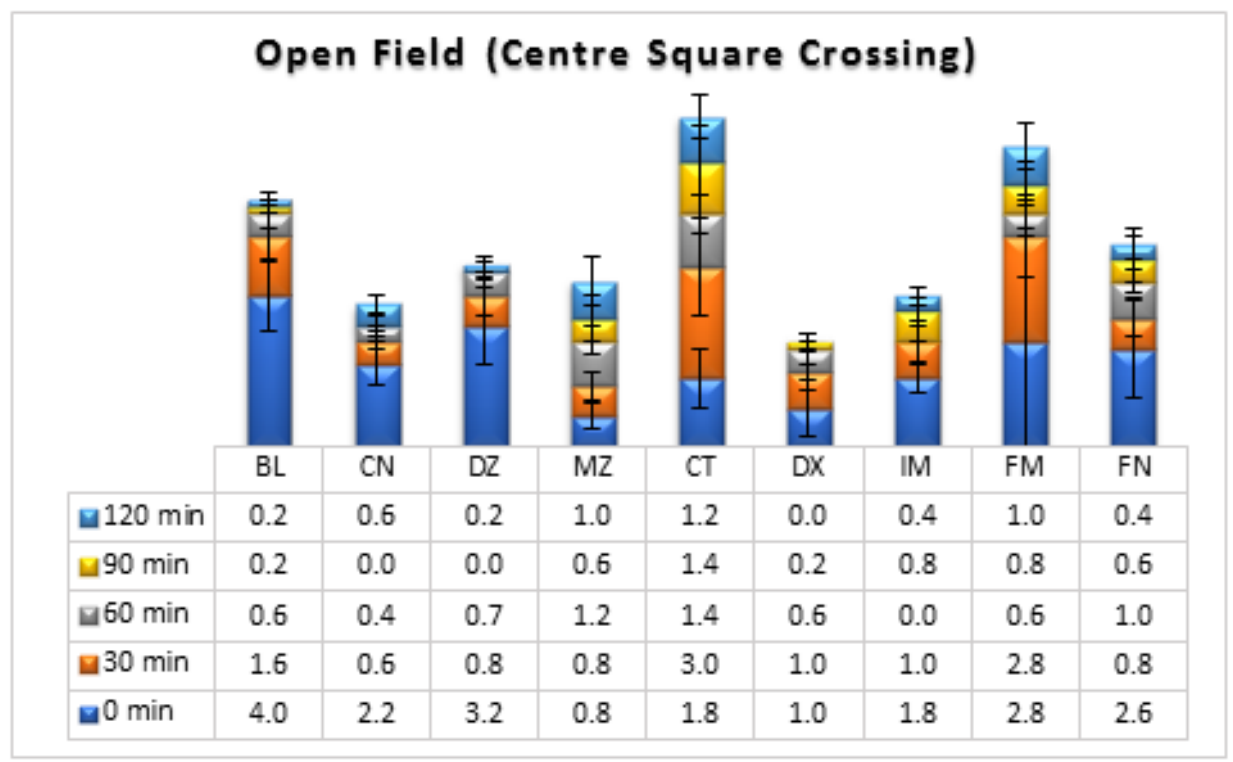

$\mathrm{BL}=$ Blank, $\mathrm{CN}=$ Control, $\mathrm{DZ}=$ Diazepam, $\mathrm{MZ}=$ Mirtazapine, $\mathrm{CT}=$ Citalopram, $\mathrm{DX}=$ Duloxetine, $\mathrm{IM}=\mathrm{Imipramine}, \mathrm{FM}=\mathrm{Flupen}$ tixol $+\mathrm{Melitracen}$, $\mathrm{FN}=$ Fluphenazine+Nortriptyline. Values are expressed as mean \pm S.E.M., $(\mathrm{n}=5)$; Dunnett $\mathrm{t}$-test $(2$ sided) treated one group as control (water) and compared all other groups against it.

Figure 6. Number of Center Square Crossing exhibited by the mice in different time intervals 


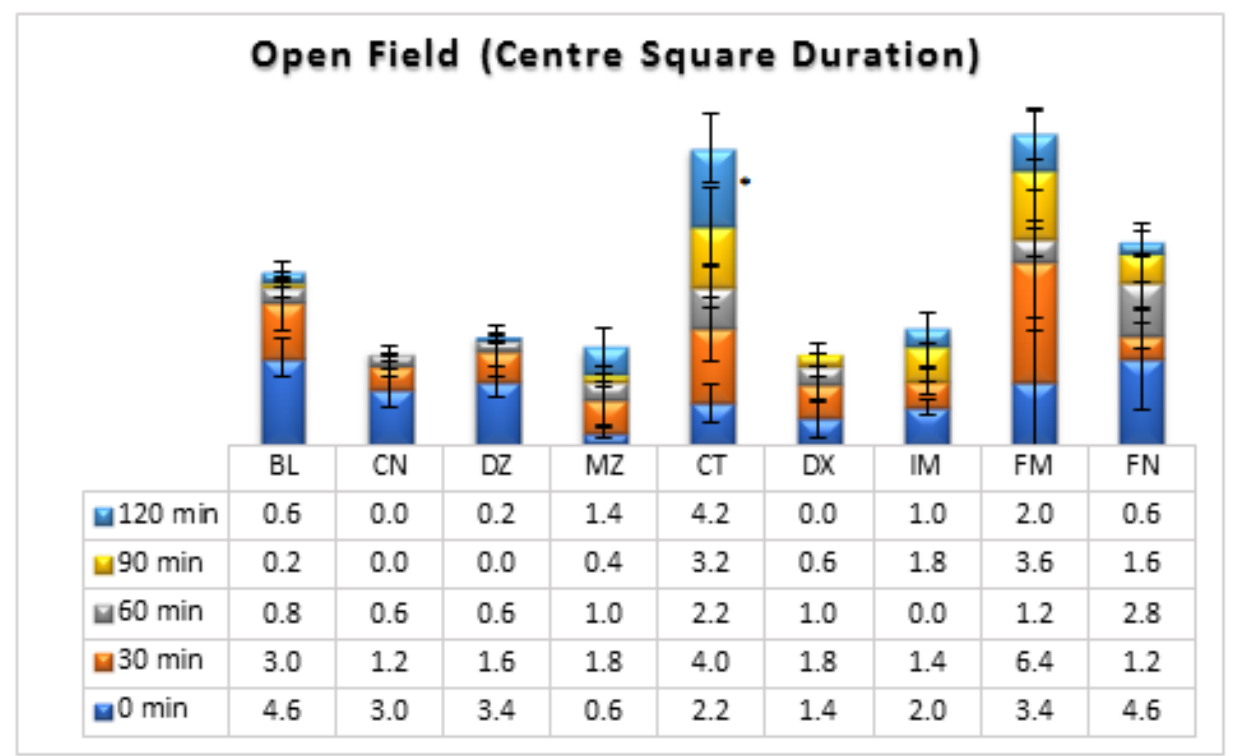

$\mathrm{BL}=$ Blank, $\mathrm{CN}=$ Control, $\mathrm{DZ}=$ Diazepam, $\mathrm{MZ}=$ Mirtazapine, $\mathrm{CT}=$ Citalopram, $\mathrm{DX}=$ Duloxetine, $\mathrm{IM}=$ Imipramine, $\mathrm{FM}=$ Flupentixol + Melitracen, $\mathrm{FN}=$ Fluphenazine + Nortriptyline. Values are expressed as mean \pm S.E.M., $(\mathrm{n}=5) ; * \mathrm{P}<0.05$; Dunnett $\mathrm{t}$-test $(2$ sided) treated one group as control (water) and compared all other groups against it.

Figure 7. Duration in Center Square exhibited by the mice in different time intervals

\subsection{Hole Cross}

Unlike the immediate response in Square crossing and rearing in open field, imipramine was observed to increase the number of hole crossing activity in $30 \& 60 \mathrm{~min}$ (Figure 8). In contrast, mirtazapine was found to reduce the activity at all intervals.

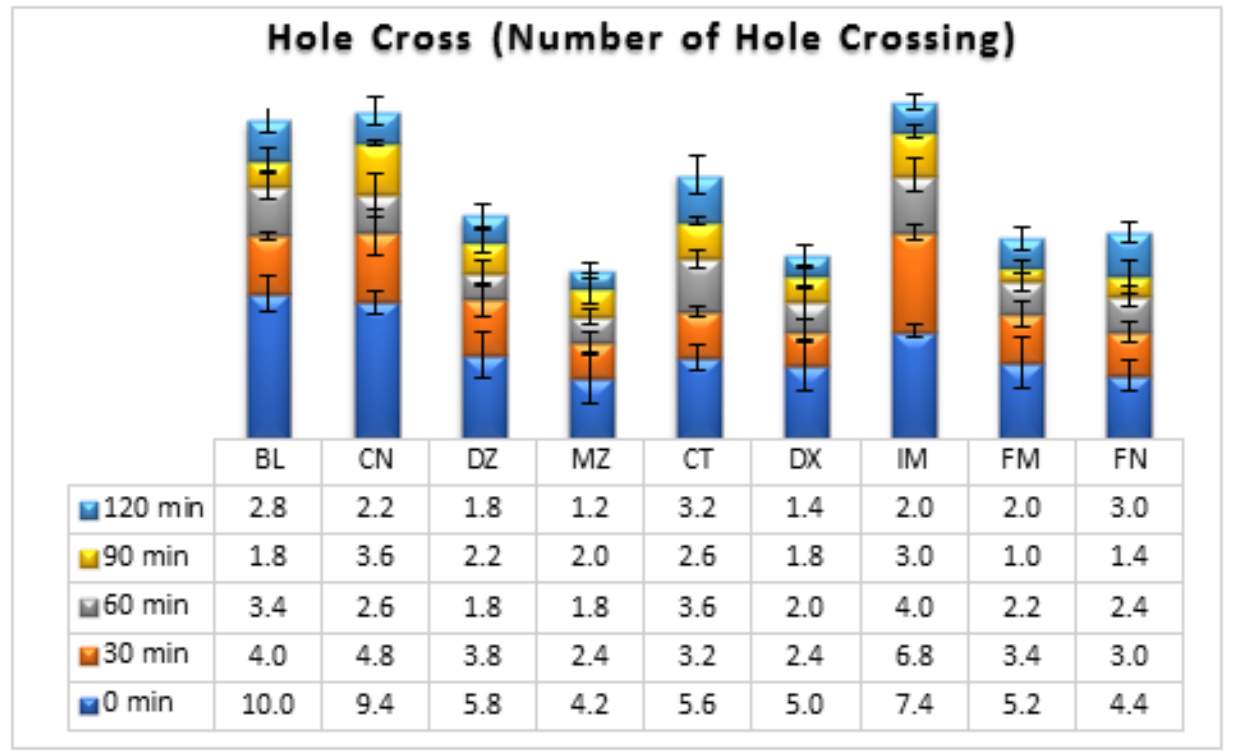

$\mathrm{BL}=\mathrm{Blank}, \mathrm{CN}=$ Control, $\mathrm{DZ}=$ Diazepam, $\mathrm{MZ}=$ Mirtazapine, $\mathrm{CT}=$ Citalopram, $\mathrm{DX}=$ Duloxetine, $\mathrm{IM}=$ Imipramine, $\mathrm{FM}=$ Flupentixol + Melitracen, $\mathrm{FN}=$ Fluphenazine+Nortriptyline. Values are expressed as mean \pm S.E.M., $(\mathrm{n}=5)$; Dunnett $\mathrm{t}$-test $(2$ sided) treated one group as control (water) and compared all other groups against it.

Figure 8. Number of Hole Crossing responses exhibited by the mice in different time intervals

\subsection{Elevated Plus Maze Test}

Figure 9 showed that duloxetine increased the entry in open arms at 60 (86.67\%) and $120 \mathrm{~min}(36.67 \%)$. Citalopram mimic the same pattern. Conversely, diazepam and the combination of flupentixol and melitracen reduced the entry mostly at $60 \mathrm{~min}$. 


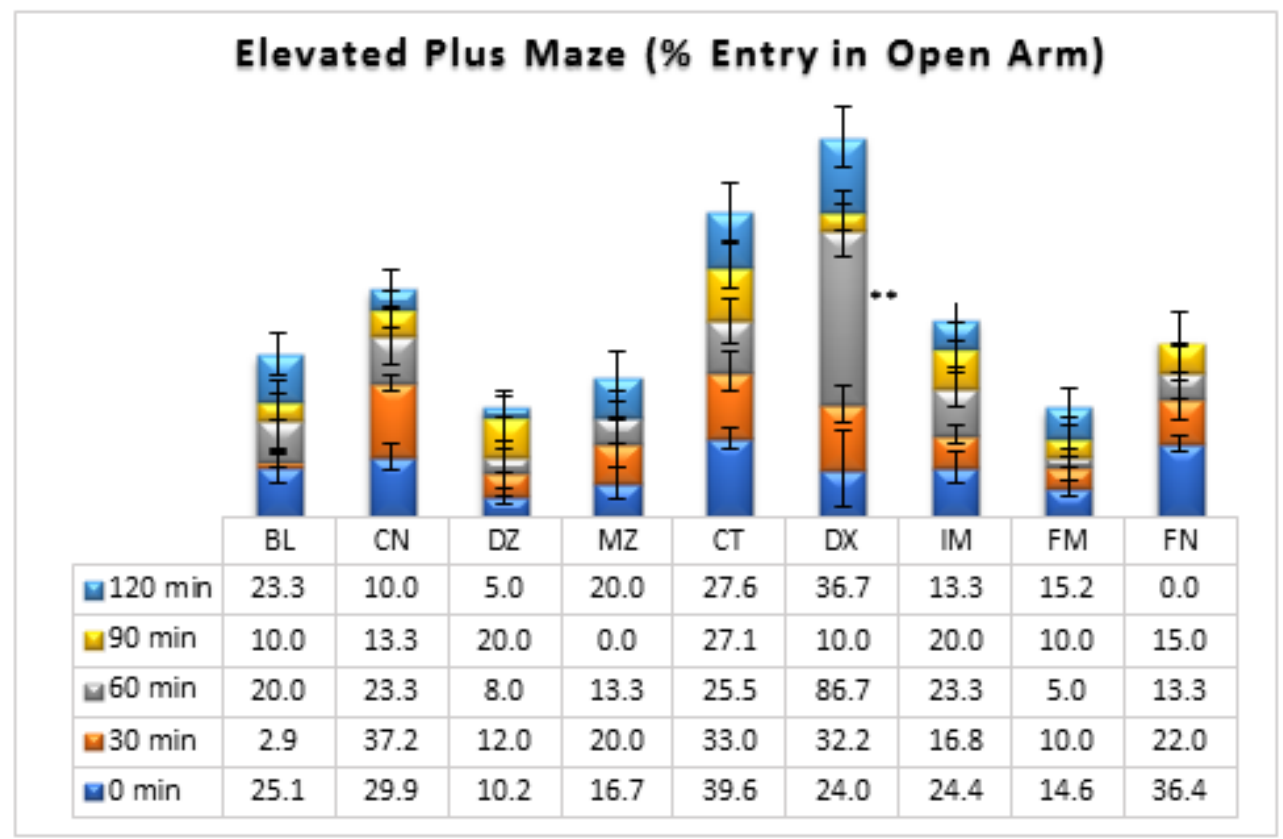

$\mathrm{BL}=\mathrm{Blank}, \mathrm{CN}=$ Control, $\mathrm{DZ}=$ Diazepam, $\mathrm{MZ}=$ Mirtazapine, $\mathrm{CT}=$ Citalopram, $\mathrm{DX}=$ Duloxetine, $\mathrm{IM}=$ Imipramine, $\mathrm{FM}=$ Flupentixol + Melitracen, $\mathrm{FN}=$ Fluphenazine+Nortriptyline. Values are expressed as mean \pm S.E.M., $(\mathrm{n}=5) ;{ }^{*} \mathrm{P}<0.05,{ }^{* *} \mathrm{P}<0.01$; Dunnett t-test $(2$ sided) treated one group as control (water) and compared all other groups against it.

Figure 9. Percentage of Entry exhibited by the mice in open arm at different time intervals

Figure 10 showed citalopram alongside duloxetine increase the duration in open arm. And then again, the combination of flupentixol and melitracen decreased the percentage of duration alongside diazepam and mirtazapine.

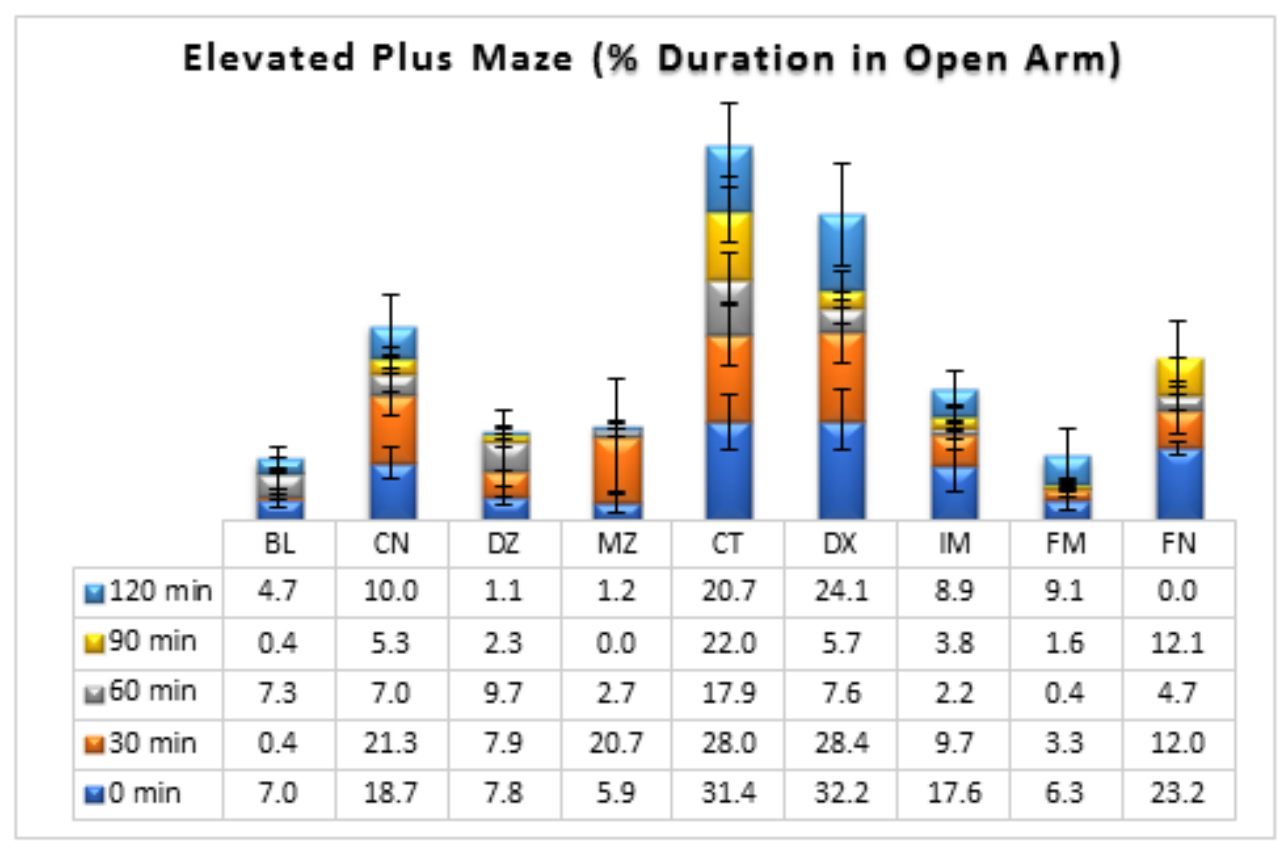

$\mathrm{BL}=$ Blank, $\mathrm{CN}=$ Control, $\mathrm{DZ}=$ Diazepam, $\mathrm{MZ}=$ Mirtazapine, $\mathrm{CT}=$ Citalopram, $\mathrm{DX}=$ Duloxetine, $\mathrm{IM}=$ =Imipramine, $\mathrm{FM}=$ Flupentixol+Melitracen, $\mathrm{FN}=$ Fluphenazine + Nortriptyline. Values are expressed as mean \pm S.E.M., $(\mathrm{n}=5)$; Dunnett $\mathrm{t}$-test $(2$ sided $)$ treated one group as control (water) and compared all other groups against it.

Figure 10. Percentage of time spent by the mice in open arm at different time intervals 


\section{Discussion}

Open Field, Hole Cross and Elevated Plus Maze experimental models are based on innate aversion of rodents to open space [3]. Integrating these three fields allowed to utilize a particular subject for all corresponding tests so as to minimize the time and personality based differences. However, this integration may have also generated a complex neurologic condition for a single subject due to the involvement of many anxiogenic stressors like diverse test environments, multiple handling and forced feeding. Keeping this risk in consideration as well as to nullify the hypothesis regarding sole existence of any particular psychotic conditions among stress, depression and anxiety; a blank and a negative control groups were deployed which demonstrated parameters like peripheral \& central movement, grooming, rearing, stretch attend posture and freezing. Several treatment approaches were applied to treat these psychological conditions including sedatives, anxiolytics and antidepressants. Different classes of selective and non-selective neurotransmitter blockers were also tested. Finally, combined drug therapies were applied to find out the treatment approach for such anxiety based depressive behaviors.

Decrease in ambulatory activities indicates persistence of anxiety and depression [14]. However, unlike peripheral movements, increase in central area movement indicates anxiolytic response which is more evident by the increase in duration at central zone [15]. Increase in peripheral movements exhibits anxiety perhaps exploratory response which is argued by neuroscientists. Similarly, number of rearing in open field and hole crossing in a hole cross apparatus by the rodents are considered measures for both locomotion and exploratory behaviors $[4,15]$. Such behaviors are considered to have proportional relation to anxiolysis. Grooming on the other hand, demonstrates the decision-making behavior and has a negative relation to the index of high activity states [16, 17], alongside its expression as consummatory and washing activities. Reduced anxiety can also be correlated with the increase in entries and duration observed in open arms of an elevated plus maze apparatus [2]. EPM includes conflict of approach and avoidance and elements of both passive and active avoidance at the same time $(18,19)$. Moreover, a measure of stretch attend posture on open field depicts the potential of rodent for risk assessment and decision making which are indicative of anxiolysis as well [20]. On the other hand, freezing observed in open field was reported as indicator for high stressed state for rodents [16].

Diazepam increased the grooming response as development of decision-making behavior. However, the sedation effect reduced rodent's potential for risk assessment (Stretch Attend Posture), locomotion (Rearing), and mitigation of fear (Entry and Duration at Open Arm). Diazepam responded as positive allosteric modulator of the
GABAA ( $\gamma$-aminobutyric acid type A) receptor complex by binding to alpha-gamma subunit interface which increases neuronal chloride-ion influx, resulting in hyperpolarization of postsynaptic membranes [21]. The potentiation of GABAA receptor at $\alpha 2 / \alpha 3$ subunit isoforms in limbic system, thalamus, hypothalamus and cerebral cortex produce calming effect which eventually facilitates the anxiolysis process [22].

Mirtazapine produces antidepressant effect by two different mechanisms- by antagonizing the presynaptic inhibitory adrenergic alpha2-autoreceptors and alpha2-heteroreceptors as noradrenergic antidepressant; and by blocking postsynaptic 5-HT2 and 5-HT3 receptors as specific serotonergic antidepressant [23]. This facilitates anxiolysis by promoting release of norepinephrine and 5-HT1A-mediated serotonergic transmission, which can be attributed to the increased grooming response [24]. Like diazepam, mirtazapine also produced the sedative effect on mice.

Selective Serotonin Reuptake Inhibitors (SSRIs) are thought to be effective in anxiety disorders alongside depressive disorders as the group works by potentiating serotonergic activity in the central nervous system by inhibiting neuronal reuptake of serotonin $(5-\mathrm{HT})[25,26]$. Citalopram therefore, found to be increasing the ambulatory activities (square crossing, rearing, center square crossing and EPM entry \& duration), decreasing the freezing condition however, it could not significantly develop risk assessment behavior.

Anxiety associated with depression is often treated with selective 5-HT and noradrenaline re-uptake inhibitors (SNRIs) as serotonin is thought to be involved in regulating mood and sleep whereas norepinephrine regulates the fight-or-flight stress response and reactions to anxiety [27]. Duloxetine is believed to bring equilibrium back to the levels of 5-HT and noradrenaline in the synaptic cleft which prevents the re-uptake and subsequent degradation of 5-HT and noradrenaline interacting at their re-uptake transporters [28]. Unlike citalopram, duloxetine was associated with reduced ambulation but mitigated fear. Yet, it failed to produce significant decision-making and risk assessment potential for animal.

Depression has also been linked to a lack of stimulation of the post-synaptic neuron by norepinephrine and serotonin [29]. Imipramine, a tricyclic antidepressant (TCA) relieves symptoms of depression by inhibiting the neuronal reuptake of the neurotransmitters norepinephrine and serotonin [30]. Binding to the sodium-dependent serotonin transporter and sodium-dependent norepinephrine transporter it reduces the reuptake of norepinephrine and serotonin [31]. Such effects were found through increased locomotor activities both in open fields and hole cross experiments and decreased high stress level (freezing).

The rationale of using combined anxiolytics (Flupenthixol) and antidepressant drug (Melitracen) was to 
find out the treatment for novelty induced anxiety based depression. Flupenthixol exerts its anxiolytic effects by antagonizing postsynaptic dopamine receptors (D1 and D2), eventually increasing the amount of serotonin and noradrenaline in the CNS [32]. Despite the fact that the actual mechanism has not been yet reported, melitracen (TCA) is believed to reduce the re-uptake of norepinephrine and 5-HT at the synapse which results in high concentration of these neurotransmitters at the post-synaptic end [33]. The combination developed decision making and risk assessing ability and alleviation of fear was evident from its central zone movement but yet, could not show its effect on parameters of EPM.

Another combination therapy was trialed to investigate the treatment approach for unconditional stress using fluphenazine (antipsycotic) and nortriptyline (TCA) [34]. Fluphenazine works by blocking postsynaptic dopamine D2 receptors in the limbic, cortical system and basal ganglia [35] whereas nortriptyline exerts its action either by inhibiting the reuptake of the neurotransmitter serotonin at the neuronal membrane or interacting with beta-adrenergic receptors [36]. Therefore, in open field, it decreased ambulation, increased decision making and risk assessment behavior and mitigated fear.

Diazepam, duloxetine and the combination of fluphenazine and nortriptyline were also found to increase the freezing activity which can be attributed to their sedative potential and cannot be correlated with high stress level of mice as their efficacy on other parameters suggested stress relieving potential.

\section{Conclusions}

Over the past few decades, improvement in understanding of neurological behavior became a crucial need for neuroscientists. Where new method development is critical and time consuming, a new approach with the existing method to minimize the gaps can serve the unfilled demand. The strategy undertaken in the present study has been validated with standard drugs and thus this integrated approach has proved to be a useful tool for assessing anxiety related depressive behavior of rodents.

\section{Conflict of Interests}

All authors agreed on the article before submission and had no conflict of interests.

\section{Funding}

This research did not receive any specific grant from funding agencies in the public, commercial, or not-for-profit sectors.

\section{Acknowledgement}

The present study was supported and carried out in the Pharmacology lab of Institute for Pharmaceutical Skill Development and Research, Bangladesh. Authors are grateful to the institution for providing such opportunity to contribute to health science.

\section{REFERENCES}

[1] Walsh, R. N. and Cummim, R. A. (1976). The open-field test - A critical review. Psychol. Bull. 83(3): 482-504. PMID: 17582919.

[2] Rodgers, R. J. and Dalvi, A. (1997). Anxiety, defence and the elevated plus-maze. Neurosci. Biobehav. Rev.21: 801810. PMID: 9415905.

[3] Sestakova, N., Puzserova, A., Kluknavsky, M. and Bernatova, I. (2013). Determination of motor activity and anxiety-related behaviour in rodents: methodological aspects and role of nitric oxide. Interdiscip. Toxicol. 6 (3): 126-135. PMID: 24678249. DOI: 10.2478/intox-2013-002 0 .

[4] Takagi, K., Watanabe, M. and Saito, H. (1971). Studies on the spontaneous movement of animals by the hole cross test: Effect of 2-dimethylaminoethane. Its acylates on the central nervous system. Jpn. J. Pharmacol.21: 797-810. PMID: 5316865.

[5] Pellow, S., Chopin, P., File, S. E. and Briley, M. (1985). Validation of open: closed arm entries in an elevated plus-maze as a measure of anxiety in the rat. J. Neurosci. Methods.14: 149-167. PMID: 2864480.

[6] Pardon, M. C., Gould, G. G., Garcia, A., Phillips, L., Cook, M. C., Miller, S. A., Mason, P. A. and Morilak, D. A. (2002). Stress reactivity of the brain noradrenergic system in three rat strains diff ering in their neuroendocrine and behavioral responses to stress: implications for susceptibility to stress-related neuropsychiatric disorders. Neuroscience.115: 229-242. PMID: 12401336.

[7] Billah, M. M., Hasan, M. R., Nawrin, K., Mohiuddin and Habib, M. R. (2015). Evaluation of Analgesic and Sedative-anxiolytic Potential of Paderia foetida Leaf Extract. Am. J. Biomed. Sci. 7(2): 98-104. DOI: 10.5099/aj150200098.

[8] Ramos, A., Moisan, M. P., Chaouloff, F., Mormede, C. and Mormede, P. (1999). Identification of female-specific QTLs affecting an emotionality-related behavior in rats. Mol. Psychiatry.4: 453-462. PMID: 10523818.

[9] Dubovicky, M., Paton, S., Morris, M., Mach, M. and Lucot, J. B. (2007). Effects of combined exposure to pyridostigmine bromide and shaker stress on acoustic startle response, pre-pulse inhibition and open field behavior in mice. J. Toxicol.27: 276-283. PMID: 17265421. DOI: $10.1002 /$ jat. 1210.

[10] Nawrin, K., Billah, M. M., Jabed, M. S. U., Roy, A., Ahmad, A. K. M. R. and Islam, M. N. (2015). Antipyretic, Antidiabetic, Thrombolytic and CNS Depressant Potential 
of Ethanol Extract of Crotalaria Verrucosa L. Leaves. Am. J. Biomed. Sci. 7(4): 198-204. DOI: 10.5099/aj150400198.

[11] Khatoon, M. M., Khatun, M. H., Islam, M. E. and Parvin, M. S. (2014). Analgesic, antibacterial and central nervous system depressant activities of Albizia procera leaves. Asian. Pac. J. Trop. Biomed. 4(4): 279-84. PMID: 25182551. DOI: 10.12980/APJTB.4.2014C348.

[12] Verma, P., Hellemans, K. G. C., Choi, F. Y., Yu, W. and Weinberg, J. (2009). Circadian phase and sex effects on depressive/anxiety-like behaviors and HPA axis responses to acute stress. Physiol. Behav.99: 276-285. PMID: 19932127. DOI: 10.1016/j.physbeh.2009.11.002.

[13] Hawiset, T., Muchimapura, S., Wattanathorn, J. and Sripanidkulchai, B. (2011). Screening neuropharmacologic al activities of Kaempferia parviflora (Krachai dam) in healthy adult male rats. Am. J. Appl. Sci. 8(7): 695-702. DOI: 10.3844/ajassp.2011.695.702.

[14] Masood, A., Banerjee, B., Vijayan, V. K. and Ray, A. (2003). Modulation of stress induced neurobehavioral changes by nitric oxide in rats. Eur. J Pharmacol. 458: 135139. PMID: 12498917.

[15] Liebsch, G., Montkowski, A., Holsboer, F. and Landgraf, R. (1998). Behavioural profiles of two Wistar rat lines selectively bred for high or low anxiety-related behaviour. Behav. Brain Res.94: 301-310. PMID: 9722280.

[16] Ivinskis, A. (1968). The reliability of behavioural measures obtained in the open-field. Aust. J. Psychol. 20(3): 173-177. DOI: $10.1080 / 00049536808255754$.

[17] Prescott, R. (1970). Some behavioral -effects of variables which influence general level of activity of rats. Anim. Behav.18: 791-796.

[18] Montgomery, K. C. (1955). The relation between fear induced by novel stimulation and exploratory drive. J. Compar. Physiol. Psychol.48: 254-260. PMID: 13252152.

[19] Handley, S. L. and McBlane, J. W. (1993). An assessment of the elevated X-maze for studying anxiety and anxiety-modulating drugs. J. Pharmacol. Toxicol. Meth. 29: 129-138. PMID: 8103377.

[20] Ehrlich, A. and Rum, S. (1958). Exploratory behavior of the black fooled ferret. Canadian Journal of Psychology.12: 235-241. PMID: 13608338 .

[21] Nicholas, E. C. and James, C. B. (2014). Classics in Chemical Neuroscience: Diazepam (Valium). ACS. Chem. Neurosci. 5(4): 253-260. DOI: 10.1021/cn5000056.

[22] Zakusov, V. V., Ostrovskaya, R. U., Kozhechkin, S. N., Markovich, V. V., Molodavkin, G. M. and Voronina, G. T. A. (1977). Further Evidence for Gaba-Ergic Mechanisms in Action of Benzodiazepines. Arch. Int. Pharmacodyn. Ther. 229(2): 313-326. PMID: 23084.

[23] Sami, A. K. A. and Esa, V. J. L. A. (2001). A review of the pharmacological and clinical profile of mirtazapine. CNS Drug Rev. 7(3): 249-64. DOI: 10.1111/j.1527-3458.2001.t b00198.x.

[24] Fawcett, J. and Barkin, R. L. (1998). Review of the results from clinical studies on the efficacy, safety and tolerability of mirtazapine for the treatment of patients with major depression. J Affect. Disord. 51(3): 267-85. PMID:
10333982. DOI: 10.1016/S0165-0327(98)00224-9.

[25] Fish, E. W., Faccidomo, S., Gupta, S. and Miczek, K. A. (2004). Anxiolytic-like effects of escitalopram, citalopram, and R-citalopram in maternally separated mouse pups. J. Pharmacol. Exp. Ther. 308(2): 474-80. PMID: 14593091. DOI: 10.1124/jpet.103.058206.

[26] National Center for Biotechnology Information. PubChem Compound Database; $\mathrm{CID}=2771$, https://pubchem.ncbi.nl m.nih.gov/compound/2771 (accessed Mar. 7, 2019).

[27] Dell'Osso, B., Buoli, M., Baldwin, D. S. and Altamura, A. C. (2010). Serotonin Norepinephrine Reuptake Inhibitors (SNRIs) in Anxiety Disorders: A Comprehensive Review of Their Clinical Efficacy. Human Psychopharmacology. 25(1): 17-29. PMID: 20041476. DOI:10.1002/hup.1074.

[28] Rang, H. P., Dale, M. M. and Ritter, J. M. (2001). Noradrenergic transmission. In Pharmacology, 4th ed. Harcourt Publishers Ltd: Edinburgh, UK.139-163.

[29] Heninger, G. R., Delgado, P. L. and Charney, D. S. (1996). The revised monoamine theory of depression: a modulatory role for monoamines, based on new findings from monoamine depletion experiments in humans. Pharmacopsychiatry. 29(1): 2-11. PMID: 8852528. DOI: $10.1055 / \mathrm{s}-2007-979535$.

[30] Gillman, P. K. (2007). Tricyclic antidepressant pharmacology and therapeutic drug interactions updated. Br. J. Pharmacol. 151(6): 737-48. PMID: 17471183. DOI: 10.1038/sj.bjp.0707253.

[31] Drugbank, 2005. Imipramine. https://www.drugbank.ca/dr ugs/DB00458/ (accessed on 07 March, 2019).

[32] Drugbank, 2005. Flupentixol. https://www.drugbank.ca/dr ugs/DB00875 (accessed on 14 May, 2019).

[33] Medicine India. Flupenthixol + Melitracen Pharmacology. https://www.medicineindia.org/pharmacology-for-generic/ 885/flupenthixol-melitracen (accessed on May 15, 2019).

[34] Brodie, N. H., McGhie, R. L., O'Hara, H., O'Hara, J., Rodway, A. E., Valle-Jones, J. C. and Schiff, A. A. (1976). Once daily administration of a fluphenazine/nortriptyline preparation in the treatment of mixed anxiety/depressive states. Curr. Med. Res. Opin.4:5: 346-352. PMID: 795608. DOI: $10.1185 / 03007997609109327$.

[35] National Center for Biotechnology Information. PubChem Compound Database; $\mathrm{CID}=3372$, https://pubchem.ncbi.nl m.nih.gov/compound/3372 (accessed Mar. 7, 2019).

[36] National Center for Biotechnology Information. PubChem Database. Nortriptyline, CID $=4543$, https://pubchem.ncbi. nlm.nih.gov/compound/4543 (accessed on May 15, 2019). 